\title{
Electronystagmographic studies of small vertical fixation movements
}

\author{
H. F. HUDDLESTON \\ $R A F$ Institute of Aviation Medicine, Farnborough, Hants.
}

Statistical estimates of the accuracy with which electronystagmography (ENG) can provide eye movement records have been published by Shackel (1960). His data described horizontal eye movements over a fairly large displacement, as did data from trials comparing this technique with eye movement films described by Hoffman, Wellman, and Carmichael (1939) and Michael and Melvill Jones (1966). Elsewhere, Shackel and Davis ( 1960 ) demonstrated that monocular vertical potentials were only about 60 per cent. of the magnitude of binocular horizontal potentials, which inferred that a vertical eye movement may yield a signal with a poorer signal-to-noise ratio than a comparable horizontal one. In the opinion of Miles (1939), who presented no supporting data, this vertical potential may possibly be enlarged if the eyes are closed. Vertical fixation movements could still be particularly difficult to measure accurately, however, because of an eyelid artefact which can confuse the vertical eye movement record (Barry and Melvill Jones, 1965).

The aim of the present investigation was to perform a test-retest reliability examination of ENG records, when such records were made from small vertical eye movements. A comparison between the eye open - eye closed case was also included.

\section{Subjects}

32 subjects participated, nine of them female. Most had normal binocular visual acuity, none poorer than 6/12 corrected (as tested by the Snellen chart). None had any visible or known eye abnormality. Their ages ranged from $17 \cdot 4$ to $50 \cdot 3$ years, but only eight were in fact over 30 years old. All were known to the experimenter and none was unduly anxious about the experimental situation, as far as could be seen.

\section{Equipment}

ENG signals were obtained from suction-cup electrodes (described by Shackel, 1958) and delivered to d.c. amplification equipment. This equipment was of high input impedance (differential input) and low drift, and had gains, available by switching, from $\times 1,000$ to $\times 10,000$. The output was $-3 \mathrm{db}$ down at $6 \mathrm{~Hz}$, and $-44 \mathrm{db}$ at $50 \mathrm{~Hz}$. (A secondary output, flat to $60 \mathrm{~Hz}$, could be introduced as an occasional check that such steep filtering was not misleading.) The amplified signals were delivered to an ultra-violet trace recorder.

\section{Procedure}

The subjects were taken individually from their laboratories or offices (each supplied with an officially-maintained lighting level), and allowed to adapt to the (normal) laboratory illumination level for to to 12 minutes. During this time, a small area above and below the non-preferred eye (for the cup electrodes) and a larger area on the ipsilateral volar forearm (for the earth electrode 
plate) were cleaned with alcohol (dilute, 90 per cent. BP) and abraded with electrode jelly ("Cardioluxe") on a gauze swab. The cup electrodes were placed about $\mathrm{I} \cdot 8 \mathrm{in}$. apart, as facial structure allowed, to be symmetrically above and below the iris when the eye was in the straightforward position. After a brief equipment demonstration, the subjects were encouraged to relax.

Each subject was then seated, the head position being maintained by a rubber-covered metal browrest, and was informed that the experiment would be finished within 2 minutes. The experimenter asked each subject to close the non-preferred eye and then to touch the closed lid lightly over the corneal apex with the index finger of the ipsilateral hand. This procedure was repeated three or four times, by way of practice, and was aimed at reducing eyelid tremor to negligible proportions, without recourse to the use of adhesive tape (which figures in the technique of Aschan, Bergstedt, and Stahle, 1956). Two fixation marks subtending $8^{\circ}$ vertically on the rough-textured white wall normal to the subject's line of gaze were named "Top" and "Bottom" fixation points. Working illumination (290-350 lux at worktop height) was provided by fluorescent units. No additional check was made on fixation accuracy.

A small experimental design randomly assigned sixteen subjects to the experimental schedule.

Both eyes open - non-preferred eye closed - both eyes open

The other sixteen followed the complementary schedule:

$$
\text { Closed - open - closed }
$$

$5+10+5$ angular changes of $8^{\circ}$ (as per the schedule) were then recorded per subject. Fixation changes were requested verbally, at the rate of one per $\mathrm{x} \cdot 5$ seconds or so, using the simple command Top ... bottom ... top ... bottom ... etc. If the entire sequence of twenty fixations was not completed in 45 seconds, it was repeated, in an attempt to avoid spontaneous changes in ocular potential magnitude which occur over time (see, for example, Byford, 1964) and in response to illumination changes (Miles, 1940; Kris, 1958; Arden and Kelsey, 1962).

\section{Results}

Limited pre-tests showed that securing the closed lid with the index finger did apparently abolish tremor, and introduced a d.c. level shift without apparently affecting the potential output for a given angular eye movement.

Best fit straight lines were drawn through each individual fixation record, disregarding the alternate records which might normally be used to assess equipotential lines more reliably. The upper trace in the Figure makes this clear. The separation on the record was then measured for each fixation, taking any axis drift into account. These measurements could then be compared in magnitude with a $100 \mu \mathrm{V}$ calibration signal recorded from a square-wave generator.

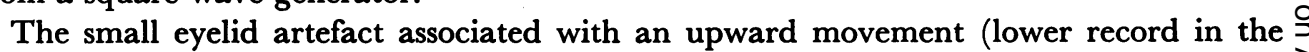
Figure, peaks at the left end of each upper fixation line) is typical of this subject. Some
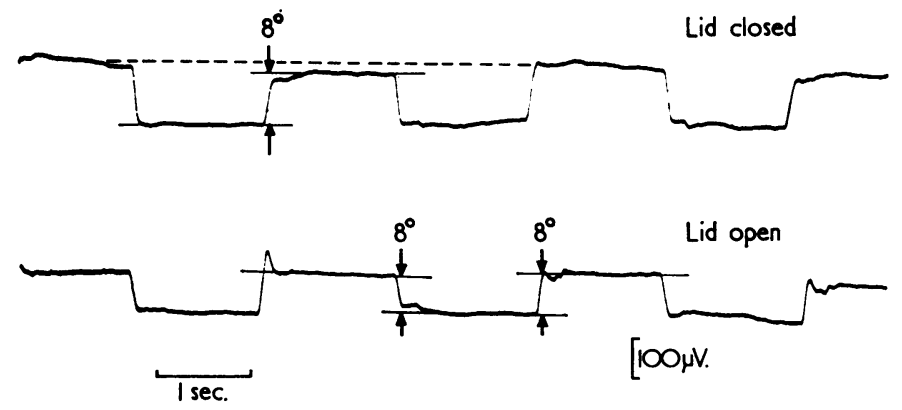

FIGURE Example electronystagmographic records, showing method of measurement. Utpper 6 record represents fixations with $\mathbb{\complement}_{2}$ the eye closed 
subjects appear to produce nearly no artefact, others a large pulse, but consistency was not systematically studied.

It was then possible to calculate, for each subject, the mean ENG potential (in $\mu \mathrm{V}$ ) produced by the ten fixations with the eye open and by the ten fixations with the eye closed. The ratio $\mu \mathrm{V}$ open $/ \mu \mathrm{V}$ closed could then be computed for each subject. Overall, there was no difference between the two conditions (as shown by the Wilcoxon $T$ statistical test: Siegel, 1956).

Using only the ten fixations forming the continuous centre section of each subject's experimental schedule, coefficients of variation could be calculated. These express the scatter (standard deviation) of the fixation record amplitudes as a percentage of the mean amplitude. The sixteen coefficients describing record variability with the eye closed were not different from the sixteen with the eye open (as shown by the Mann-Whitney $U$ statistical test: Siegel 1956), and could therefore be pooled. This confirms the observation that inconsistencies such as that illustrated in the top trace of the Figure occur equally with the eye open and with the eye closed.

The results are given in Table I. Distributions were apparently normal for all three data sets, but the data population is, of course, too small to permit a statistical test of this.

Table I Electronystagmography. Reliability and potential magnitude data

\begin{tabular}{|c|c|c|c|}
\hline Measurement & Mean & Median & Range \\
\hline Potential $\left(\mu \mathrm{V} / 8^{\circ}\right)$ eye open & 96 & 87 & $3^{8-189}$ \\
\hline Potential ratio $\frac{\mu \mathrm{V} \text { eye open }}{\mu \mathrm{V} \text { eye closed }}$ & $1 \cdot 09$ & $\cdot 97$ & $\cdot 4-2 \cdot 1$ \\
\hline $\begin{array}{l}\text { Coefficient of variation } \\
\text { 100 S.D. }\end{array}$ & & & \\
\hline Mean & $11 \cdot 20$ & $10 \cdot 70$ & $4 \cdot 8-23 \cdot 7$ \\
\hline
\end{tabular}

Note: A high coefficient indicates unreliability

Using the Wilcoxon $T$ statistic (two tails) it was shown that the group of 32 subjects could be analysed as in Table II.

Table II Potential magnitude, comparing eye open and eye closed, for 32 subjects

\begin{tabular}{|c|c|c|c|c|c|c|}
\hline \multicolumn{3}{|c|}{ Greater potential for eye open } & \multirow{2}{*}{$\begin{array}{l}\text { No } \\
\text { difference }\end{array}$} & \multicolumn{3}{|c|}{ Greater potential for eye closed } \\
\hline$P<\cdot 01$ & $\mathbf{P}<\cdot 02$ & $P<\cdot 05$ & & $P<\cdot 05$ & $\mathrm{P}<\cdot 02$ & $\mathbf{P}<\cdot 01$ \\
\hline I I & o & 2 & 7 & $\mathbf{I}$ & 2 & 9 \\
\hline
\end{tabular}

This breakdown was independent of experimental schedule.

Spearman rank order correlations (as described by Siegel, 1956) were used to confirm $\left(\mathrm{P}<\cdot 0 \mathrm{I} ; r_{s}=0.503\right)$ that larger potential magnitudes permitted greater reliability of measurement. It was further confirmed that age is no useful predictor of ocular potential magnitude $\left(r_{s}=-0 . I_{1}\right.$, insignificant: Shackel, $196 \overline{6}$, mentions his own and others' work leading to the same conclusion). Nor was age related to the potential ratio $\mu \mathrm{V}$ open $/ \mu \mathrm{V}$ closed $\left(r_{s}=-0.09 \mathrm{I}\right.$, insignificant $)$. Tests using the Mann-Whitney $U$ 
statistic (Siegel, I956) showed that the older sixteen subjects (containing all ten with less $\frac{\vec{J}}{3}$ than normal visual acuity) were insignificantly different from the younger sixteen subjects $\underline{0}$ in terms of the parameters in Table I.

\section{Discussion}

When a random sample of the general population is required for accurate ENG recording, $\frac{\bar{p}}{\vec{D}}$ ENG potential magnitude, and hence record measurement accuracy will not be improved $\stackrel{\mathbb{Q}}{\varrho}$ by the closure of the eye. Individuals may be selected from the population, however, for a potential reliably greater for either the eye open or the eye closed case.

The eyelid movement artefact which can confuse ENG records from the vertical axis $\overrightarrow{\vec{H}}$ can, of course, be suppressed by closing the eye. Requesting individuals to touch the ${ }_{S}^{\omega}$ closed lid of their non-preferred eye eliminates the artefacts due to lid tremor without confounding the ENG record.

In the more reliable 68 per cent. (mean \pm I SD) of an average, short record (less than $45 \mathrm{sec}$.) the eye yields an ENG signal representing anything from $7 \cdot 1^{\circ}$ to $8.9^{\circ}$ from an $v$ initial locus for a requested rotation of $8 \cdot 0^{\circ}$. The error can be in excess of $3.5^{\circ}$ for a few을 individuals, however. Little of this can be referable to the normal eye movements of ${ }_{c}^{-}$ fixation (Ditchburn and Foley-Fisher, 1967), although some may be due to inaccurate fixation. Outside this more reliable data, very large errors are occasionally seen which are surely not due to faulty fixation, but more likely to the vagaries of the ocular potential. Casual inspection of the records suggests that errors operate randomly, such that the $\vec{\oplus}$ arithmetical average of ten to twenty measurements might be expected to be highly. reliable indeed.

\section{Summary}

Electronystagmographic (ENG) records were taken from 32 individuals required to $\stackrel{\circ}{\mathbb{Q}}$ perform $8^{\circ}$ vertical fixations. Estimates were made of ENG record reliability. Closing $\overrightarrow{\hat{O}}$ the eye eliminates any eyelid movement artefact, of course, but does not generally affect ${ }^{\Im}$ the ENG potential level which goes to determine record measurement accuracy.

\section{References}

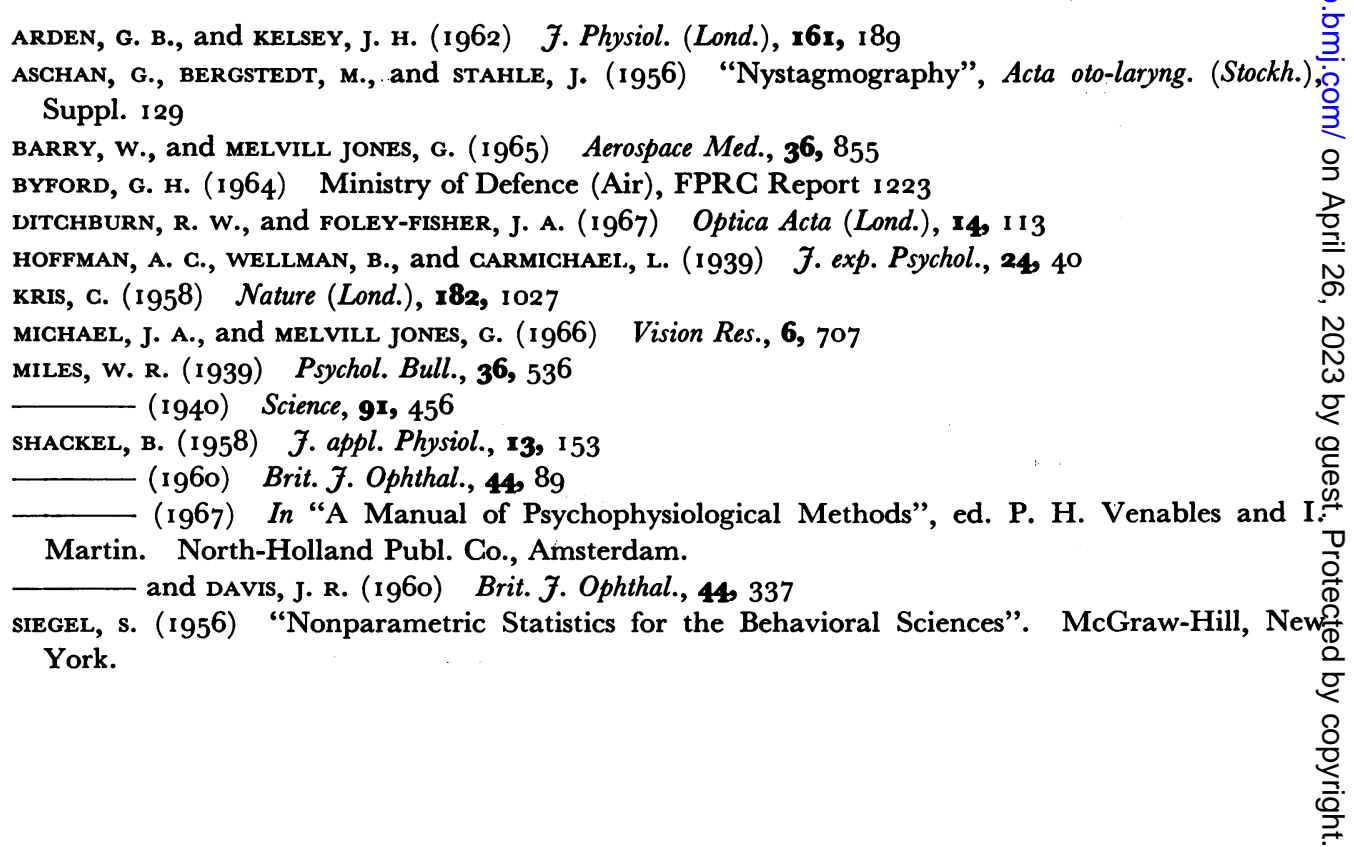

